\title{
Article
}

\section{Intelligent Discrimination Method Based on Digital Twins for Analyzing Sensitivity of Mechanical Parameters of Prestressed Cables}

\author{
Zhansheng Liu ${ }^{1,2, *(D)}$, Guoliang Shi ${ }^{1,2}$, Antong Jiang ${ }^{1,2}$ and Wenjie Li ${ }^{1,2}$ \\ 1 Faculty of Architecture, Civil and Transportation Engineering, Beijing University of Technology, \\ Beijing 100124, China; shiguoliang@emails.bjut.edu.cn (G.S.); jiangat@emails.bjut.edu.cn (A.J.); \\ liwj0827@emails.bjut.edu.cn (W.L.) \\ 2 The Key Laboratory of Urban Security and Disaster Engineering of the Ministry of Education, \\ Beijing University of Technology, Beijing 100124, China \\ * Correspondence: lzs4216@163.com
}

Citation: Liu, Z.; Shi, G.; Jiang, A.; Li, W. Intelligent Discrimination Method Based on Digital Twins for Analyzing Sensitivity of Mechanical Parameters of Prestressed Cables. Appl. Sci. 2021, 11, 1485. https://doi.org/10.3390/ app11041485

Academic Editors: Hugo Filipe Pinheiro Rodrigues and Ivan Duvnjak

Received: 22 December 2020

Accepted: 3 February 2021

Published: 6 February 2021

Publisher's Note: MDPI stays neutral with regard to jurisdictional claims in published maps and institutional affiliations.

Copyright: (c) 2021 by the authors. Licensee MDPI, Basel, Switzerland. This article is an open access article distributed under the terms and conditions of the Creative Commons Attribution (CC BY) license (https:/ / creativecommons.org/licenses/by/ $4.0 /)$.

\begin{abstract}
The information collected on large-span prestressed cables by field sensors is susceptible to interference, which leads to inaccurate collection of structural and mechanical parameters of large-span prestressed cables, resulting in misjudgment of structural safety performance. This paper proposes an intelligent judgment method for improving the sensitivity of analyzing mechanical parameters of prestressed cables based on digital twins (DTs). The safety performance of the structure was evaluated by analyzing the mechanical parameters. First, the information during prestressed cable tensioning is dynamically sensed, thereby establishing a multidimensional model of structural analysis. The virtual model is processed by the model modification rule to improve the robustness of the simulation; thus, a DT framework for the sensitivity judgment of the mechanical parameters of the cable is built. In the twin model, the simulation data of the real structure were extracted. Probabilistic analysis was performed using the Dempster-Shafer(D-S) evidence theory to discriminate the sensitivity of mechanical parameters of each cable node under the action of external forces with high accuracy and intelligence. Sensitivity analysis provides a reliable basis for the safety performance assessment of structures. Taking the wheel-spoke-type cable truss as an example, the application of DTs and D-S evidence theory to the sensitivity determination of cable mechanical parameters under temperature fully verified that the proposed intelligent method can effectively evaluate the safety performance of the actual structure.
\end{abstract}

Keywords: digital twin; Dempster-Shafer evidence theory; prestressed cable; sensitivity of mechanical parameter; intelligent discrimination; structural safety assessment

\section{Introduction}

With improvements in construction technology, the use of prestressed steel structures is widespread. With superior strength, flexible shape, and being light weight, prestressed space structures are commonly used in large public buildings [1]. In recent years, they have mostly been used in sports stadiums in China. The quality of construction of large-span spatial structures is also an important measure of a country's construction technology and capability, and the mechanical properties of the cables directly determine the overall performance of the structure [2]. Because large-span spatial structures are mostly used in buildings of high importance and large quantities are involved in the construction process, the safety requirements of the construction process are strict. Many experts and scholars have conducted a lot of research on the safety control of prestressed steel structures in the tensioning process.

Chen et al. [3] conducted research on a new type of tensioning construction forming method, construction error influence, and control technology for cable domes called the 
divisional lifting overall tensioning method. The application of this method during construction avoids large member displacement, thus improving the safety of construction. Guo et al. [4] investigated the effect of the initial cable length error in the prestressing state on the sensitivity of prestressed cables to length error. By controlling the length error, the prestress level during cable tensioning was effectively improved. Zhang et al. [5] proposed a joint-square double-brace structure to improve the force performance of the spatial structure and derived a formula for calculating the prestressing of the members of the structure under their self-weight, which provided theoretical support for the stability verification of the structure during the construction process. Xue et al. [6] studied a single-story saddleshaped cross-cable network without an inner ring. By studying the nonlinear analysis of the forces of the structure based on the raw-dead unit method, the structural resistance to the continuous collapse of the inner-loop cable network was effectively improved. Liu et al. [7] used the response surface method and Monte Carlo method to calculate the structural reliability index and obtain the effect of tensioning cable relaxation at different locations on structural reliability, which allows the calculation of prestress loss in spatial structures and provides a basis for the safety assessment of structures. Asadolahi, SM. et al. [8] proposed equations to estimate the joint stiffness and energy dissipation reduction of the system by analyzing it for two time periods, during and after the application of prestress. Arezki et al. [9] investigated the effect of temperature variation on the safety performance of cable truss structures and cables. Castillo et al. [10] derived the damage accumulation process of a cable system under normal loading and established a theoretical model for the fatigue life of a cable considering load redistribution. Shekastehband et al. [11] conducted a theoretical analysis and experimental study on the sudden breakage of cables in tensioned monolithic structures, pointing out that severe cases can cause a continuous collapse of the tensioned monolithic structure.

There are several shortcomings in the component sensitivity discrimination and safety performance assessment of prestressed steel structures in the above studies. (i) The safety assessment of structures relies on data collected by field sensors, resulting in insufficient accuracy of structural performance analysis. (ii) The damage level of the structure cannot be displayed visually, resulting in insufficient information for structural performance analysis. With the development of the new generation of information technology and the promotion of industrial information systems, the application of technologies such as Digital twins (DTs) to engineering construction has become an increasingly researched topic [12]. Applying DTs to engineering practice can significantly improve the accuracy and intelligence of structural performance analysis.

DT technology is a technique that simulates the state and behavior of physical entities portrayed in a dynamic virtual model with high fidelity [13]. Zhang et al. [14] combined DTs and virtual reality technology to propose an intelligent control method for underground equipment, which solved the problem of difficulty in remotely controlling cantilevered road heading machines. Gong et al. [15] proposed an intelligent regulation and control system for air flow at the outlet of the digging face by analyzing the DT technology, thereby realizing the mapping interaction between the physical entity and the virtual twin in the coal mining process. Meng et al. [16] used DT technology in vehicle life prediction and maintenance decision making, which describes the working state of the system more accurately, thus enabling online optimal decision making and feedback control of the vehicle. Szpytko et al. [17] proposed an integrated maintenance decision model for cranes under operating conditions based on the DT concept, which effectively improved the operational efficiency of cranes and reduced the safety risk of equipment operation. Zhou et al. [18] proposed a DT model of a centrifugal impeller and established parametric centrifugal impeller DTs, which can significantly improve the design and manufacturing level of the centrifugal impeller and shorten the design cycle. Yu et al. [19] proposed a DT framework for solving the health state monitoring problem of complex systems, which improves the accuracy and reduces the uncertainty of analysis for the health monitoring of complex systems in intelligent manufacturing. Krishnan et al. [20] used the DT concept for 
the detection of electric drive faults, which improved the performance analysis accuracy of electric vehicles during usage. Seon et al. [21] conducted a study to accurately analyze the structural integrity and remaining service life of aircraft. Based on the study of highfidelity nondestructive inspection techniques, a DT of the structure was created to optimize the monitoring mechanism, which provided a reference for the performance analysis of complex structures. This shows that DT technology has been studied and widely applied in manufacturing.

Owing to the lack of research on the assessment of structural safety performance during tensioning of prestressed steel structures and combined with the status of research on DTs, this paper proposes an intelligent discrimination method based on DTs for the sensitivity analysis of mechanical parameters of prestressed cables. The safety performance of the structure is intelligently evaluated through the analysis of the sensitivity of the component mechanical parameters. First, based on the concept of DTs, a framework for sensitivity discrimination was established, and the real tension was dynamically perceived and simulated. In the virtual model, the mechanical parameters of the structure can be extracted directly, and the mechanical properties of the structure can be displayed intelligently. After extracting the mechanical parameters, the Dempster-Shafer (D-S) evidence theory was fused to process the structural mechanical performance information. Therefore, the change probability of the mechanical parameters of each node of the cable was accurately analyzed, and the sensitivity of the prestressed cable was intelligently determined, thus providing a basis for the safety performance evaluation of the structure. Driven by DTs, the D-S evidence theory is integrated to realize intelligent discrimination of the sensitivity of the mechanical parameters of the prestressed cable. Based on the above theoretical method, application was made to a wheel-spoke cable truss as an example. In this study, the sensitivity of the mechanical parameters of the cable under the effect of temperature during tensioning was analyzed, and the effectiveness of this method was verified.

\section{Construction of a DTs Framework for Sensitivity Discrimination}

Prestressed steel structures have the highest safety risk during the construction phase because of the incompleteness of the structure, time-varying nature of the material, complexity of the loads applied, and immaturity of the structural resistance. Mechanical analysis during cable tensioning is in the domain of construction mechanics, where the geometric, physical, and boundary parameters are functions of time. The safety assessment of the tensioning process is a multidimensional mechanical problem of the coupling time and space. Combined with the characteristics of tensioning safety assessment, this study considers the sensitivity of the mechanical parameters of tension cables as an entry point. The reliability of the structure is determined by analyzing the sensitivity of the mechanical parameters at each point in the member. Supported by the concept of DTs, the dynamic perception and simulation of realistic tension are performed, and the mechanical performance state of the structure is intelligently displayed by the twin model.

\subsection{Capture of Twin Information}

The DTs replicate a real physical entity through visual virtual space modeling, and the virtual model simulates the dynamic behavior of that entity in a real environment [22,23]. It is necessary to compare multidimensional structural data during assessment of the structural safety state [24]. Therefore, the first step in the twin modeling of cable tensioning is to identify the factors influencing the safety for analysis. The changes in the external effect and structural mechanical properties of the prestressed steel structure subjected to the tensioning process are the external and internal causes of the structural state change. The changes in the structure and mechanical performance of the structure can directly change the state of the structure. Likewise, the changes in the structural state of the prestressed steel structure in the tensioning process will also cause a change in the structural mechanical properties; thus, the changes in the structural state can be reflected in the degree of changes 
in the structural mechanical properties. The state of the structure directly responds to the safety performance of the structure. The intrinsic relationship between the basic factors influencing the safety performance of prestressed steel structures during tensioning is shown in Figure 1.

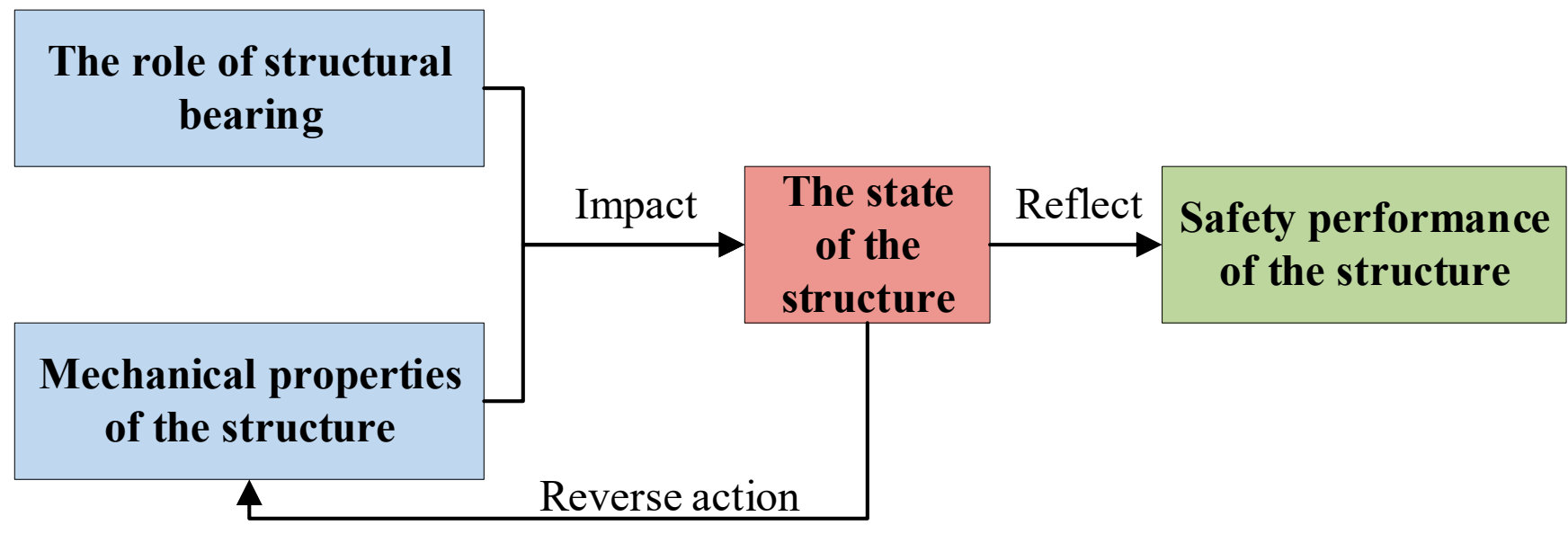

Figure 1. Intrinsic relationship of basic influencing factors of safety performance of prestressed steel structures during tensioning.

From the intrinsic relationship of the factors influencing the structural safety performance, it can be concluded that the information to be captured for the tensioning process is mainly divided into the action to which the structure is subjected (S) and the mechanical properties of the structure (R). The twin model established in this study can accurately map the state of the structure.

1. The role of structure bearing

There are multiple causes of structural damage or destruction during the tensioning process. By combining the effects of various external influences [25], this study focuses on capturing and sensing the member length errors $\left(L_{e}\right)$, wind load effects $\left(W_{l}\right)$, and temperature effects $\left(T_{e}\right)$. The component length error is the most important factor causing the initial form and pretension deviation of the cable tension structure [26]. The effective control of the length error effect is key in the design of prestressed steel structures, especially in the design of construction tensioning. In an actual project, the geometric error of the cable tension structure mainly includes the error of the release length of the cable, error of the size of the anchorage or node, and deviation of the bearing position. Geometric errors can cause deviations in the initial form of the flexible pretensioned structure design, resulting in inconsistencies between construction and design and a consequent loss in prestress. The severity and sensitivity of the wind vibration effects of large-span space structures are no less than those of high-rise and towering structures. In most cases, wind loads tend to be controlling loads in the design of structures [27]. The cable is a flexible system, which very easily deforms under the wind load, thus affecting the safety performance of the entire structure. Therefore, the monitoring of wind pressure helps to scientifically predict the safety performance of cables. Because steel structures have temperature-sensitive properties, the analysis of temperature effects is very important in the design and construction of large-span steel structures [28]. Changes in temperature result in temperature stresses and temperature deformations in the structure. Prestressed cables are important elements of superstationary structures, and the effect of temperature inevitably causes changes in the internal forces of the cables, thus affecting the safety performance of the structure.

2. Mechanical properties of the structure

It is more common to determine the safety performance of a structure according to the state of the prestressed steel structure during tensioning. The state of the structure refers to 
all the forms of the structure and its materials, and the state of the structure is influenced by the mechanical properties of the structure. Based on the mechanical properties of the cables, the sensitivity of the mechanical parameters at each point can be discerned, and the reliability of the structure can be assessed. The key elements in the analysis of prestressed steel structures during tensioning are the mechanical property indices, such as cable force $\left(C_{f}\right)$, deflection $(\omega)$, stress $(\delta)$, strain $(\varepsilon)$, and crack $(C)$, which are closely related to the reliability of the construction process of the structure.

\subsection{Multidimensional Modeling for Intelligent Discrimination of Sensitivity to Mechanical Parameters of Cables}

In this study, the structural information of cable tensioning is derived from the finite element model of the structure, which is highly integrated with each construction element of the prestressed steel tensioning process and contains sufficient twin data to support the analysis of the sensitivity of the member mechanical parameters. This allows an intelligent analysis of the safety performance of structures. Moreover, finite element analysis has been widely used as a better mechanical simulation tool in the structural construction industry [29]. The twin information is divided into two categories based on the analysis of the information that needs to be captured for tension safety assessment: the action to which the structure is subjected and the mechanical properties of the structure. These are expressed by Equation (1) and Equation (2). As important simulation information for the twin model, the action on the structure $(S)$, and the mechanical properties of the structure $(R)$ are expressed specifically as:

$$
\begin{gathered}
S=\left(L_{e}, W_{l}, T_{e}\right) \\
R=\left(C_{f}, \omega, \delta, \varepsilon, C\right)
\end{gathered}
$$

In Equation (1), $L_{e}$ denotes the member length errors, $W_{l}$ denotes the wind load effects, and $T_{e}$ denotes the temperature effects. In Equation (2), $C_{f}$ denotes the cable force, $\omega$ denotes deflection, $\delta$ denotes stress, $\varepsilon$ denotes strain, and $C$ denotes a crack.

During the creation of the twin model, the action to which the structure is subjected and the mechanical properties of the structure are collected in real time using sensors. At the same time, a virtual model of the real structure is created during the finite element analysis. In the virtual model, the twin data of the effect of the structure and the mechanical properties of the structure are simulated by setting the corresponding working conditions. This enables the twin simulation of the realized tensioning process and establishes a model basis for the intelligent discrimination of the sensitivity of the mechanical parameters of the prestressed cables driven by the DTs. This in turn provides a basis for the analysis of the safety performance of structure construction. A multidimensional model for the intelligent discrimination of the sensitivity of the mechanical parameters of the cables is represented by Equation (3). The multidimensional model consists of five dimensions, expressed as follows:

$$
D T M=\left(S_{p r}, S_{v m}, L_{d p}, L_{f a}, C_{n}\right)
$$

In Equation (3), DTM denotes a multidimensional model for the intelligent discrimination of the sensitivity of the mechanical parameters of the cables, $S_{p r}$ denotes the physical structure entity, $S_{v m}$ denotes the virtual structure model, $L_{d p}$ denotes twin data processing layer, $L_{f a}$ denotes the functional application layer, and $C_{n}$ denotes the connection between the components. The multidimensional twin model enables the simulation and mapping of realistic tensioning processes. By processing the structural parameters from realistic monitoring and virtual simulation, the sensitivity of the mechanical parameters of the structural components can be determined. As a result, the safety performance of the structure can be assessed, and precise maintenance of the construction site can be achieved. The multidimensional model for the intelligent discrimination of the sensitivity of the mechanical parameters of the oriented cables is shown in Figure 2. 


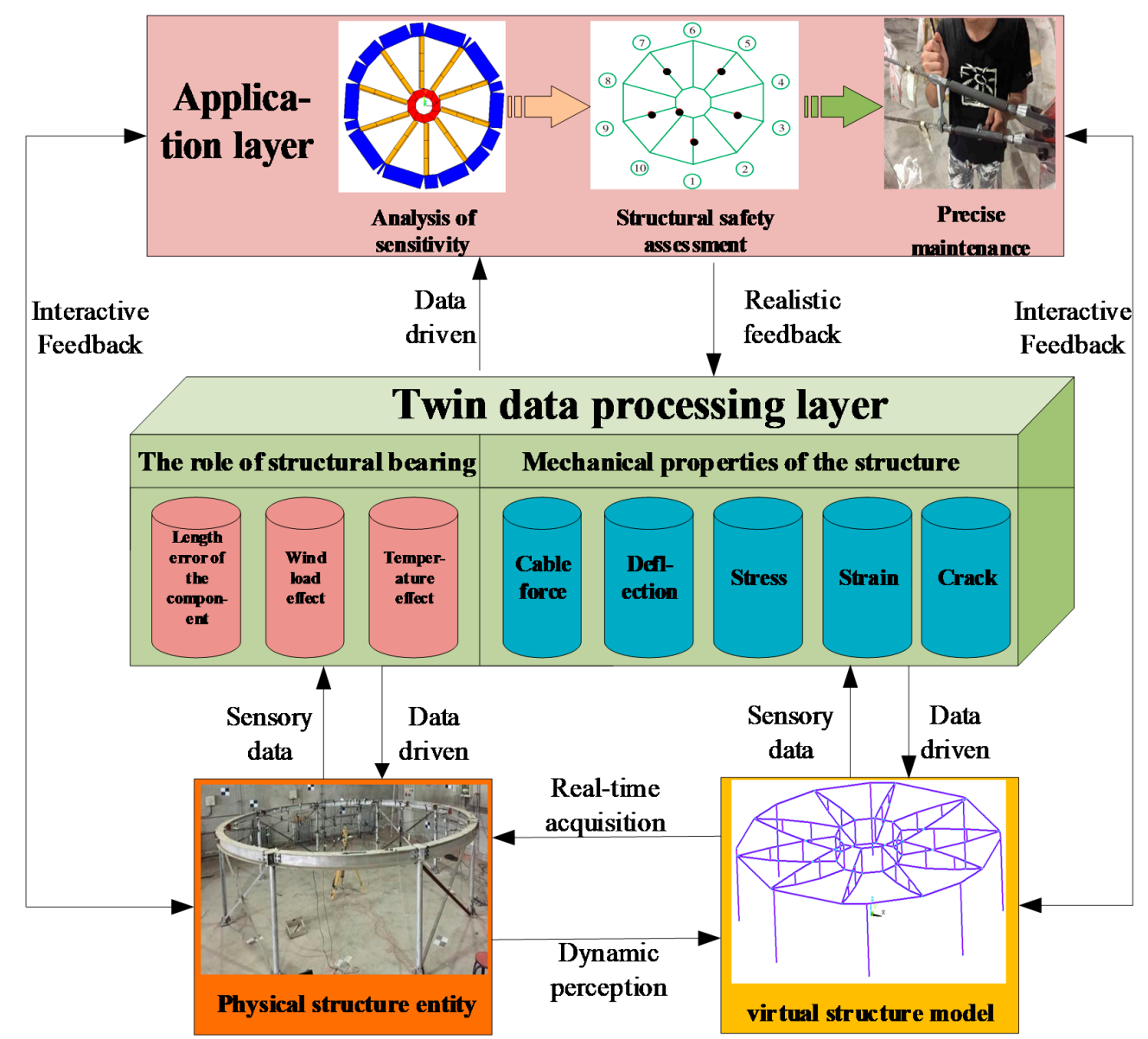

Figure 2. Multidimensional model for intelligent discrimination of sensitivity to mechanical parameters of cables.

\subsection{Correction of the Virtual Model}

Under the condition of establishing a multidimensional model, to enhance the simulation capability of the virtual model, it is necessary to overcome the high fidelity of the model and correct the virtual twin model of the tensioning process of the cables. The average weighting method [30] was used to fuse the monitored data of realistic tensioning and the simulated data of the finite element model to correct the virtual model. The basic principle involves selecting different weights for different location sensitivity indicators to achieve the goal of minimizing the sum of squared Euclidean distances between the fusion results and each sensitivity indicator.

Suppose that the actual monitored values of a mechanical parameter at each node of a cable in a certain working condition are $x_{1}, x_{2}, \ldots, x_{n}$. Then, the average value $\bar{x}$ of the actual monitored values is expressed as:

$$
\bar{x}=\sum_{i=1}^{n} x_{i} / n
$$

Then the weight $\omega_{i}$ of each mechanical parameter is represented by Equation (5), which is expressed as:

$$
\omega_{i}=\left(\frac{1}{d_{i}}\right) / \sum_{i=1}^{n}\left(\frac{1}{d_{i}}\right)
$$

In Equation (5), $d_{i}$ is the Euclidean distance from each mechanical parameter to the mean of the mechanical parameters, expressed as:

$$
d_{i}=\left\|\bar{x}-x_{i}\right\|
$$


From this, the weighted average of the monitored values of mechanical parameters $(\hat{x})$ for the entire cable can be calculated by combining the data of each node, that is,

$$
\hat{x}=\sum_{i=1}^{n} \omega_{i} x_{i}
$$

The overall monitoring value $D$ of the structure can be calculated from the weighted average of the monitoring values of the mechanical parameters of each cable, that is:

$$
D=\mu+\alpha_{c} \sigma
$$

$\alpha_{c}$ is the confidence level of the mechanical parameter analysis, 1.5 in this study. $\mu$ and $\sigma$ denote the mean and standard deviation of the weighted average of the monitored values of the mechanical parameters $(\hat{x})$ for each cable, respectively.

Similarly, using the above steps, the simulation values $D^{*}$ of the mechanical parameters in the finite element model can be calculated. Using Equation (9) to judge the fidelity of the simulation model, the correction of the virtual model is completed, which ensures that the simulation data effectively represent the mechanical properties of the real structure:

$$
E_{D}=\frac{\left|D^{*}-D\right|}{D} \times 100 \%
$$

In Equation (9), $E_{D}$ is a metric for determining the fidelity of the twin model.

\subsection{DTs-Driven Sensitivity Intelligence Discriminative Framework}

By establishing a high-fidelity virtual model, it is possible to simulate the mechanical properties of cables in a virtual space for realistic tensioning processes. The simulation is carried out in the virtual model for the conditions in the real tensioning process to obtain highly consistent mechanical property information with the real structure. To improve the sensitivity analysis accuracy of the mechanical parameters of the cables, multiple types of simulation data were analyzed by integrating the D-S evidence theory. The sensitivity of the mechanical parameters of each node on the cable can be intelligently discerned to provide a basis for the safety performance assessment of the structure, which in turn ensures the feasibility of the maintenance of the real structure. The intelligent discriminative framework for the sensitivity of the mechanical parameters driven by DTs is shown in Figure 3.

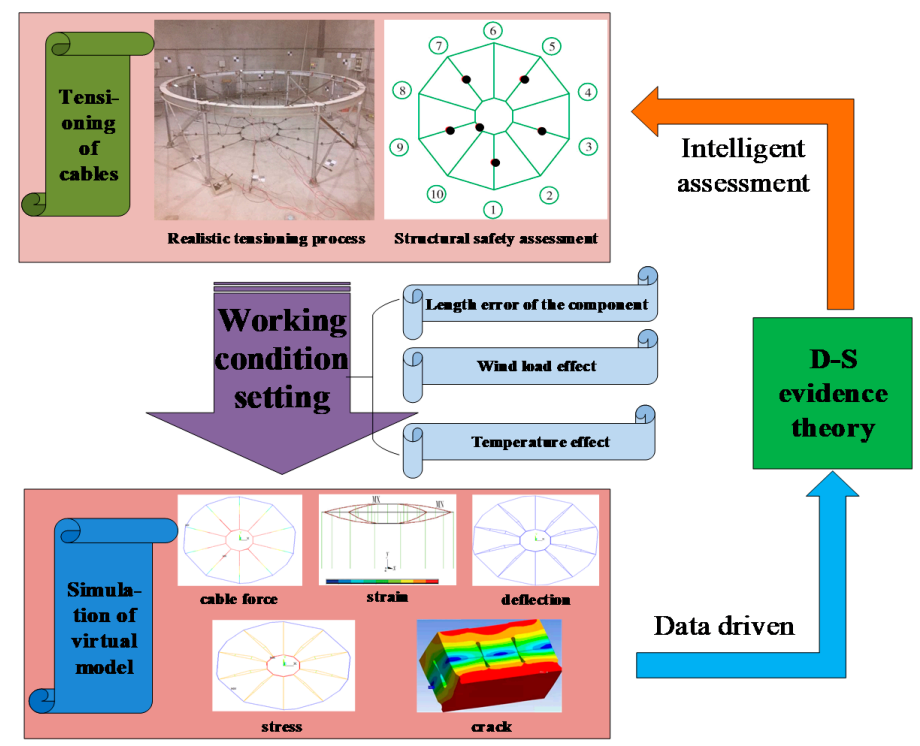

Figure 3. Intelligent discriminative framework for mechanical parameter sensitivity driven by digital twins. 


\section{Analysis of Simulation Data Based on D-S Evidence Theory}

By establishing a high-fidelity twin model, the simulated data in the model can be directly extracted for analysis, which realizes intelligent discrimination of the sensitivity of the cable mechanical parameters based on the DTs. By integrating the D-S evidence theory to process the information about the structural mechanical properties, the probability of mechanical property change at each point of the cables is calculated to discern the sensitivity of mechanical parameters at each point and provide a basis for the safety performance assessment of the structure. D-S theory, as a technique for the fusion of multisource information, belongs to the category of artificial intelligence [31] and has been applied in business administration and road transportation. In this study, it was applied to discriminate the sensitivity of the mechanical parameters of a structure to improve the accuracy of the safety assessment.

\subsection{D-S Evidence Matrix}

The basic principle of the D-S evidence matrix is as follows: based on multiple sources of heterogeneous data, multiple mechanical parameter sensitivity discriminators are identified independently, and the basic probability assignment function is determined by the probability assignment of each mechanical parameter sensitivity discriminator. The final discriminatory results are obtained by fusion using D-S synthesis rules [32].

Assume that $\Omega$ is the discriminative framework for the sensitivity of the mechanical parameters of the prestressed cable members, which is expressed by Equation (10), and the specific expression is:

$$
\Omega=\left(e_{1}, e_{2}, \cdots, e_{n}\right)
$$

In Equation (10), $e_{i}(i=1,2, \ldots, n)$ expresses the change in the mechanical properties of the $i$ th node of the prestressed cable.

All possible working conditions of the prestressed cable element are the power set " $2 \Omega$ ", which is expressed as Equation (11):

$$
2^{\Omega}=\left(\varnothing, e_{1}, e_{2}, \cdots, e_{n}, e_{1} \cup e_{2}, e_{i} \cup e_{j} \cup \cdots \cup e_{k} \cdots\right)
$$

In Equation (11), $\varnothing$ indicates no change in the mechanical properties of the prestressed cable, and $e_{i}$ indicates a change in the mechanical properties of a single node of the prestressed cable. $e_{i} \cup e_{j} \cup \cdots \cup e_{k}$ indicates the change of mechanical properties of multiple nodes of the prestressed cable.

The basic probability distribution function (mass function) can be expressed as $\mathrm{m}: 2^{\Omega}$ $\rightarrow[0,1]$, satisfying Equation (12):

$$
\left\{\begin{array}{c}
m(\varnothing)=0 \\
\sum_{A \subset \Omega} m(A)=1
\end{array}\right.
$$

In Equation (12), $A$ is a certain working condition of the prestressed cable, and $m(A)$ is the basic probability distribution function of $A$.

Assume that $m_{j}\left(A_{i}\right)$ is the basic probability distribution function for the $j$ th mechanical parameter sensitivity indicator. To determine the change in the mechanical properties of the ith node, the synthesis rule of the D-S evidence matrix is Equation (13):

$$
D F(A)=m_{1} \oplus m_{2} \oplus \cdots \oplus m_{n}(A)\left\{\begin{array}{c}
\frac{\sum_{\cap A_{i}=A} \prod_{1 \leq j \leq n} m_{j}\left(A_{i}\right)}{K}(A \neq \varnothing) \\
0(A=\varnothing)
\end{array}\right.
$$

In Equation (13), $K=\sum_{A_{1} \cap A_{2} \cap \cdots \cap A_{n} \neq \varnothing} m_{1}(A)_{1} m_{2}(A)_{2} \cdots m_{n}(A)_{n}$ $=1-\sum_{A_{1} \cap A_{2} \cap \cdots \cap A_{n}=\varnothing} m_{1}\left(A_{1}\right) m_{2}\left(A_{2}\right) \cdots m_{n}\left(A_{n}\right)$. 


\subsection{Analysis Process of Sensitivity}

The DT framework is integrated with the D-S evidence theory to intelligently discriminate the sensitivity of the mechanical parameters of the cables. First, a twin model was built for a realistic tensioned structure, and the model was modified by the weighted average method to improve the fidelity of the model. In the virtual model with high fidelity, the working conditions corresponding to the construction site were set, and the mechanical performance index data simulated in the model were extracted. The relevant information of the index data is determined probabilistically, and the sensitivity of the integrated mechanical parameters of each node of the cable is calculated by integrating the D-S evidence theory algorithm. In this study, the sensitivity of the mechanical parameters is characterized by the degree of change in the mechanical parameters of each node on the cable. By sorting the probability of change in mechanical parameters at each point of the cable, the change degree of mechanical properties at each point can be determined. According to the sensitivity of the mechanical parameters, the most vulnerable position of the structure can be directly determined, thus providing a basis for the safety performance evaluation and maintenance of the structure. The process of safety performance assessment and maintenance of the structure through a sensitivity analysis of the mechanical parameters is shown in Figure 4.

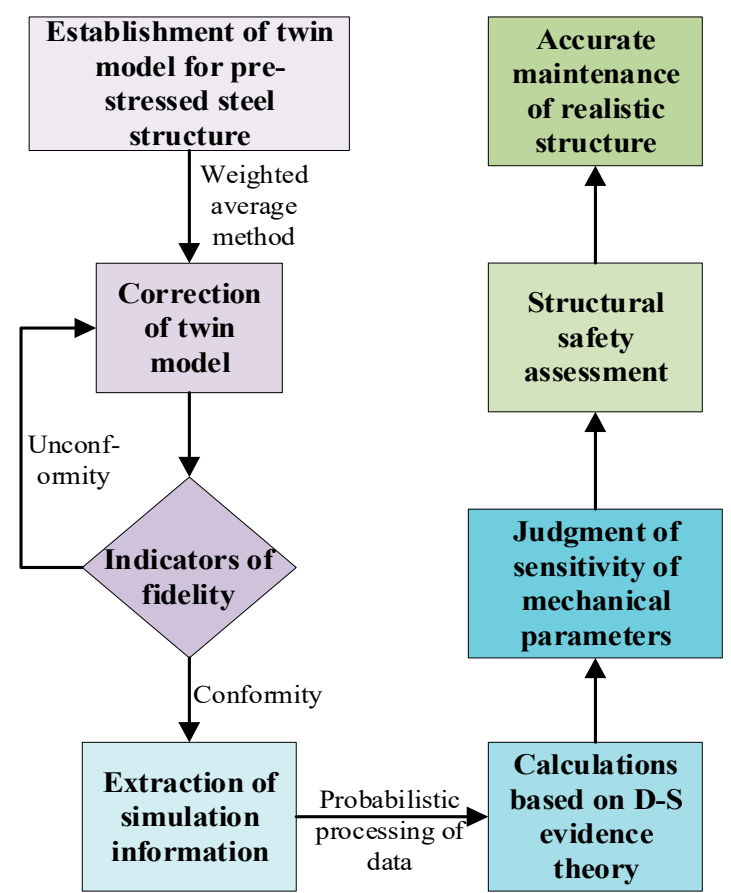

Figure 4. Safety performance assessment and maintenance process for structures.

\section{Intelligent Discrimination of the Sensitivity of Mechanical Parameters of Prestressed Cables under the Effect of Temperature}

In the intelligent discrimination framework of mechanical parameter sensitivity driven by DTs, a high-fidelity virtual model of a realistic tensioning structure is established. The twin data are also processed by integrating the D-S evidence theory, which can accurately discriminate the sensitivity of the mechanical parameters of each prestressed cable node under the effect of an external environment using model simulation data. The experimental model built in this study is a reduced-scale test model based on a certain wheel-spoke cable truss project. Compared with the actual project, the scale ratio of the test model was 1:10, cross-sectional area ratio of the cable was 1:100, and materials were identical. The structure span of the test model was $6 \mathrm{~m}$ and consisted of 10 radial cables, ring cables, braces, nodes, outer ring beams, and steel columns. The radial cables include upper and 
lower radial cableshe radial cables include upper and lower radial cables, and the ring cables include upper and lower ring cables.

The struts included the outer, middle, and inner struts. The model used to test the wheel, spoke cable truss, is shown in Figure 5. This study focuses on the analysis of changes in structural mechanical parameters under the effect of temperature difference. First, the mechanical parameters of the structure are simulated in the high-fidelity virtual model, and the degree of change in the mechanical parameters of each node is analyzed by fusing the D-S evidence theory to find and monitor the most sensitive position, thus realizing the intelligent evaluation of the safety performance of the structure. In the test process, to simulate roof installation after the cable is formed, the effect of temperature difference on the structural safety performance of the cable is considered, and the extreme working conditions are considered. Combined with the temperature change in the region where a practical project is located, it is concluded that the annual temperature change in the region is $55{ }^{\circ} \mathrm{C}$. In order to simulate the state of the real structure, a test model is built. According to the constructed test model, the working condition was set to a temperature rise of $55^{\circ} \mathrm{C}$. The initial temperature value of the structure is given first, and then the final temperature value of the structure is set by adjusting the temperature so that the temperature change is $55^{\circ} \mathrm{C}$. At the same time, the temperature change is set to increase by $1{ }^{\circ} \mathrm{C}$ per minute, and the mechanical properties of the structure are analyzed when the temperature increases by $55^{\circ} \mathrm{C}$, thus achieving consistency with realistic structure state. Sensors are arranged in the field for real-time monitoring, whereas the virtual model combined with the D-S evidence theory discriminates the sensitivity of the mechanical parameters of the cables under the effect of temperature.

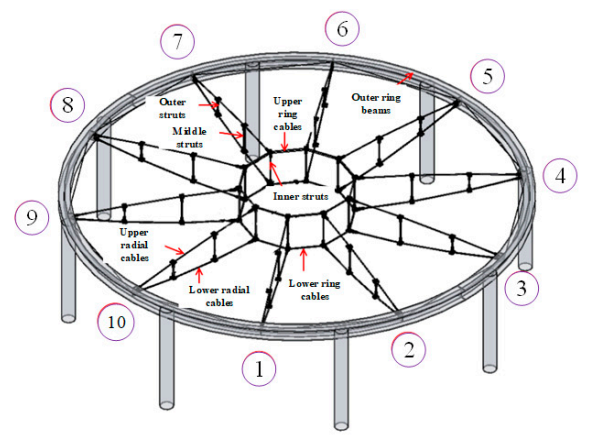

(a) Building information model of test

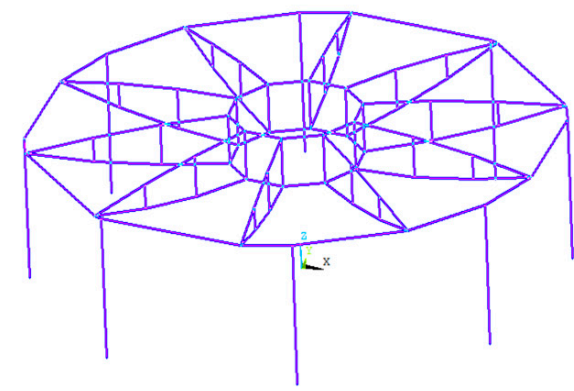

(b) Finite element model of test

Figure 5. Model for the test of the wheel-spoke cable truss.

\subsection{The Analysis Process of Sensitivity}

A high-fidelity twin model is the basis for the intelligent discrimination of the sensitivity of mechanical parameters in cables under the effect of temperature. In this study, ANSYS was used to establish a finite element model for the simulation and analysis of structural performance. In the finite element model, the mechanical performance indices such as cable force $\left(C_{f}\right)$, deflection $(\omega)$, stress $(\delta)$, strain $(\varepsilon)$, and crack $(C)$ of the structure can be simulated by setting various types of effects to which the structure is subjected. Among them, the main effects of the structure include the length error of the member $\left(L_{e}\right)$, wind load action $\left(W_{l}\right)$, and temperature action $\left(T_{e}\right)$.

After the finite element model is established, to achieve a realistic mapping of the real structure, the model needs to be modified to improve the fidelity of the model. The monitored data of the field sensors and the simulated data of the model were fused according to the model correction law proposed in Section 2.3. Finally, the parameters and node connections of the twin model were adjusted using the cable force as the control object. In the performance analysis of this structure, the analysis focused on the support and midspan position of each section of the cable. Under the self-weight of the structure, cable force sensors are arranged at the supports and midspan positions of each section 
of the upper and lower radial cables of the test structure to collect the cable forces of the cable members. The cable forces of the corresponding nodes of the cables are simulated in the twin model. In this case, the nodes of the real structure with the sensors are the same as the nodes of the simulated data in the model. Taking the upper radial cable of the first bay as an example, the nodes where the sensors are arranged in the real structure and the nodes simulated in the model are shown in Figure 6. A total of seven nodes were selected in upper radial cable 1 . The cable force and strain sensors are arranged at each control node, while the real-time acquisition of vertical displacement is carried out by a total station. In the finite element model, the analysis of the corresponding mechanical parameters is carried out for each node. Using the average weighting method, the index $\left(E_{D}\right)$ for adjusting the fidelity of the twin model is within 5\%, and mapping to the real structure can be achieved.

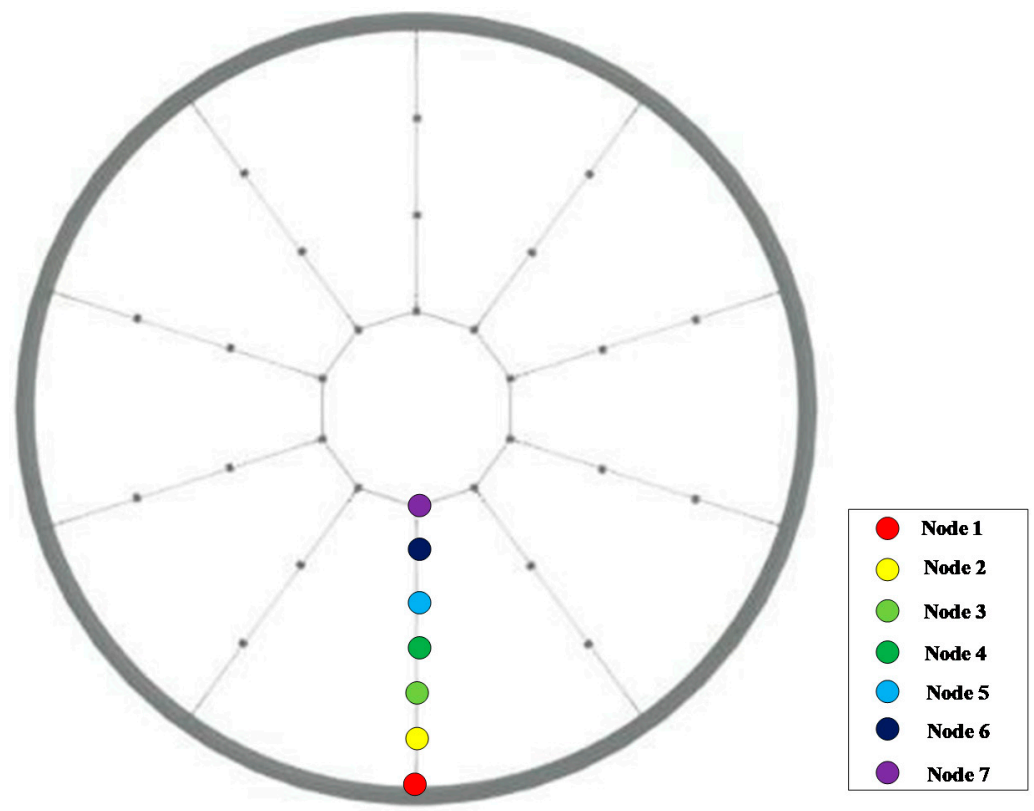

Figure 6. Schematic diagram of the selection of nodes.

In this study, the finite element model was modified by adjusting the cross-sectional area of the cable before studying the effect of temperature on the mechanical properties of the structure. In Figure 6, by collecting the cable force of each node under the action of self-weight, the weighted average method is used for data fusion. Considering that the change of the section size of the component will cause the change of the self-weight of the structure, the fidelity of the finite element model is improved by modifying the section area of the component. The dimensions of the modified finite element model members are listed in Table 1. Taking the whole of each cable as the object of examination, a comparison of the simulation of the cable force before and after the model correction is shown in Table 2.

Table 1. Dimensions of the modified finite element model members.

\begin{tabular}{ccc}
\hline Member Type & Location & Cross-Sectional Area $\left.\mathbf{( m m}^{\mathbf{2}}\right)$ \\
\hline \multirow{2}{*}{ Radial cable } & Upper level & 24.6 \\
& Lower level & 33.3 \\
Ring cable & Upper circle & 24.6 \\
& Lower circle & 49.1 \\
& Outer circle & 62.8 \\
Strut & Mid circle & 62.8 \\
& Inner circle & 62.8 \\
Ring beam & Outer circle & 4300 \\
\hline
\end{tabular}


Table 2. Comparison of simulation degree of cable force before and after model correction.

\begin{tabular}{|c|c|c|c|c|c|}
\hline $\begin{array}{l}\text { Component Unit } \\
\text { Number }\end{array}$ & $\begin{array}{c}\text { Monitoring Values of } \\
\text { the Structure } \\
(\mathbf{N})\end{array}$ & $\begin{array}{c}\text { Simulation Value } \\
\text { before Correction } \\
\text { (N) }\end{array}$ & $\begin{array}{l}\text { Simulation Value } \\
\text { after Correction } \\
\text { (N) }\end{array}$ & $\begin{array}{l}\text { Error before } \\
\text { Correction }\end{array}$ & $\begin{array}{l}\text { Error after } \\
\text { Correction }\end{array}$ \\
\hline Upper radial cable 1 & 4890 & 5572 & 4775 & $14 \%$ & $-2 \%$ \\
\hline Upper radial cable 3 & 5850 & 5563 & 5713 & $-5 \%$ & $-2 \%$ \\
\hline Upper radial cable 5 & 5150 & 5570 & 5087 & $8 \%$ & $-1 \%$ \\
\hline Upper radial cable 7 & 5660 & 5569 & 5772 & $-2 \%$ & $2 \%$ \\
\hline Upper radial cable 9 & 5140 & 5573 & 5137 & $8 \%$ & $0 \%$ \\
\hline Lower radial cable 1 & 4260 & 4481 & 4136 & $5 \%$ & $-3 \%$ \\
\hline Lower radial cable 3 & 4790 & 4473 & 4851 & $-7 \%$ & $1 \%$ \\
\hline Lower radial cable 5 & 4100 & 4569 & 4134 & $11 \%$ & $1 \%$ \\
\hline Lower radial cable 7 & 4490 & 4368 & 4655 & $-3 \%$ & $2 \%$ \\
\hline Lower radial cable 9 & 4210 & 4481 & 4302 & $6 \%$ & $2 \%$ \\
\hline
\end{tabular}

From the comparison before and after the model correction, it can be seen that the revised model can reflect the state of the real structure effectively. The data of multiple nodes of each cable are fused based on the average weighting method in the correction process to ensure that the assurance index of the virtual model is within $5 \%$. Therefore, the error of the corrected simulation is lower when each cable is studied as a whole. In this study, it can be concluded from Table 1 that the error between the simulated data of the modified model and the actual collected data is within 3\%. The resulting finite element model can realistically and accurately map the performance changes in the structure.

\subsection{Data Analysis Based on D-S Evidence Theory}

Based on the establishment of a high-fidelity finite element model, the data from the simulation of multiple mechanical parameters of the structure were fused. The sensitivity of the mechanical parameters at each node of the cable under the effect of temperature was analyzed using the probabilistic treatment and D-S evidence theory. In this study, to verify the accuracy of the judgment based on the D-S evidence theory, the points where the simulated data are collected in the model are set to be the same as the locations of the monitored points arranged in the real cables. Upper radial cable 1 is the object of study, and there are seven monitored and simulated points arranged at the support and midspan positions of each section of the cable. The selection of the monitored and simulated points is the same as that in Figure 6.

In the finite element model, a condition consistent with the real tensioning process was set, that is, a temperature rise of $55{ }^{\circ} \mathrm{C}$. The simulated values of the cable force, strain, and deflection at each node were extracted from the model, and the sensitivity of the mechanical parameters at each point under the effect of temperature was calculated by probabilistic processing. In calculating the probability of the simulated values at each point, the analysis using the D-S evidence theory should ensure that the sum of the probabilities of parameter changes at each node is 1 when a single parameter is used as the discriminant.

For example, when using the cable force as the basis for discrimination, the probability of the change in the cable force at each node is calculated using Equation (14).

$$
P_{i}^{*}=\frac{\left|C_{f i}-C_{f i}^{*}\right|}{C_{f i}}
$$

In Equation (14), $C_{f i}$ indicates the value of the cable force at each node on the cable before the effect of temperature, $C_{f i}^{*}$ and indicates the value of the cable force at each node on the cable after a temperature increase of $55^{\circ} \mathrm{C}$. To ensure that the sum of the damage probability of each node on the cable is 1 when the cable force is used as the basis for discrimination, the probability calculated by Equation (14) needs to be corrected. The 
probability $\left(P_{i}\right)$ of the change in the cable force at each node after correction was calculated according to Equation (15):

$$
P_{i}=\frac{P_{i}^{*}}{\lambda}
$$

In Equation (15), $\lambda$ denotes the coefficient of the correction of probability $\lambda=\sum_{i=1}^{7} P_{i}^{*}$. The probabilities calculated accordingly can be used in the fusion analysis of the D-S evidence theory to discriminate the sensitivity of the mechanical parameters of each node.

Similarly, the probability of change in the mechanical parameters at each point when strain and deflection are used as discriminators can be derived. Combining the D-S evidence theory to fuse various types of data and synthesize the sensitivity of each node can lead to the response of the cable to the effect of temperature. At the same time, the rate of change of the cross-sectional area of each point on the real structure is used as an important basis for the detected results of structural safety performance. The data collection of the rate of change of the cross-sectional area of each node of the cable in the field is shown in Figure 7.

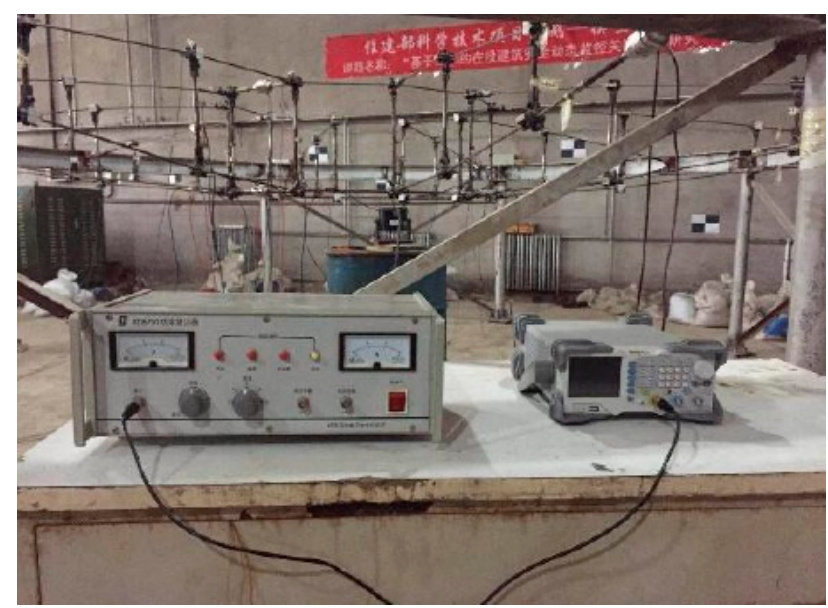

Figure 7. Data acquisition of the rate of change of cross-sectional area.

The rate of change of the cross-sectional area of each node on the cables was calculated according to Equation (16). To make the comparison of the results clearer, this study adjusted the sum of the detection result values to 1 for each point. The rate of change of the cross-sectional area of each node on the cable was corrected according to Equation (17), and the results of the detection of the structural safety performance expressed by the corrected rate of change of the cross-sectional area of each node on the cable were used for comparison with the results of the D-S evidence theory. The discrimination of the sensitivity of each node of the cable is presented in Table 3.

$$
D_{m i}^{*}=\frac{\left|A_{m i}-A_{m i}^{d}\right|}{A_{m i}}
$$

Table 3. Judgment of the sensitivity of each node of the cable.

\begin{tabular}{cccccc}
\hline Location & $\begin{array}{c}\text { Discrimination Based } \\
\text { on Cable Force }\end{array}$ & $\begin{array}{c}\text { Discrimination } \\
\text { Based on Strain }\end{array}$ & $\begin{array}{c}\text { Discrimination } \\
\text { Based on } \\
\text { Deflection }\end{array}$ & $\begin{array}{c}\text { Discriminative } \\
\text { Results of D-S } \\
\text { Evidence Theory }\end{array}$ & $\begin{array}{c}\text { Detected Results of } \\
\text { Structural Safety } \\
\text { Performance }\end{array}$ \\
\hline Node 1 & $12.5 \%$ & $12.3 \%$ & $11.2 \%$ & $8.21 \%$ & $8.69 \%$ \\
Node 2 & $13.1 \%$ & $12.8 \%$ & $12.3 \%$ & $9.84 \%$ & $9.24 \%$ \\
Node 3 & $13.7 \%$ & $13.7 \%$ & $12.7 \%$ & $11.37 \%$ & $10.72 \%$ \\
Node 4 & $15.6 \%$ & $15.9 \%$ & $14.9 \%$ & $17.63 \%$ & $18.67 \%$ \\
Node 5 & $16.4 \%$ & $15.6 \%$ & $15.6 \%$ & $19.04 \%$ & $20.18 \%$ \\
Node 6 & $14.9 \%$ & $15.3 \%$ & $16.9 \%$ & $18.38 \%$ & $19.43 \%$ \\
Node 7 & $13.8 \%$ & $14.4 \%$ & $16.4 \%$ & $15.53 \%$ & $14.68 \%$ \\
\hline
\end{tabular}


In Equation (16), $D_{m i}^{*}$ denotes the rate of change of the cross-sectional area of each node of the cable in a realistic structure, and $A_{m i}$ denotes the cross-sectional area of each node on the cable before the effect of temperature. $A_{m i}^{d}$ denotes the cross-sectional area of the nodes on the cable after a temperature increase of $55^{\circ} \mathrm{C}$.

$$
D_{m i}=\frac{D_{m i}^{*}}{k}
$$

In Equation (17), $D_{m i}$ denotes the corrected rate of change of the cross-sectional area of each node on the cable, and $\mu$ indicates the coefficient of correction of the rate of change, $k=\sum_{i=1}^{7} D_{m i}^{*}$.

In Table 3, by comparing the change rate of mechanical parameters of each point based on D-S evidence theory analysis and the change rate of area based on field acquisition, it can be obtained that when the temperature increases by $55{ }^{\circ} \mathrm{C}$, the order of sensitivity of each point is consistent, and the sensitivity of node 5 is the highest, so it is necessary to focus on monitoring the node.

\subsection{Analysis of Experimental Results}

In the modification of the finite element model, it is possible to effectively target a structural parameter as the control object; however, the other simulated parameters are not necessarily guaranteed to be consistent with the real structure. As the change in several mechanical parameters will have an impact on the cross-sectional area of the components and each mechanical parameter is also taken into consideration when probing the safety performance of the real structure, it is necessary to make a comprehensive judgment on the various types of data from the simulation by means of data fusion. The probability of mechanical property change at each node of the cable can be determined more precisely by the D-S evidence theory incorporating various sensitivity discriminant parameters. By comparing the detected results with the safety performance of the real structure, the ranking of the damage degree of each node is consistent, and the synchronization of the structural sensitivity degree of each point analyzed with the real monitoring value is above $95 \%$, which provides a reliable basis for the safety assessment of the structure. In this study, with a temperature increase of $55^{\circ} \mathrm{C}$ as the working condition, it was determined that the most sensitive position of the mechanical parameters of upper radial cable 1 under the effect of temperature is at node 5 . This needs to be closely monitored to ensure the reliability of the structure.

To verify the validity of the proposed method, the fidelity of the model and the number of discriminative indicators were varied in this study, and experimental analyses were performed on other cables as well. During the test, the mechanical parameters of each cable were analyzed. In this paper, the upper radial cable 1 is described as the key analysis object. The average weighting method is used to modify the virtual model, whereas the D-S evidence theory is used to integrate various types of discriminant indices for the sensitivity analysis of structural mechanical parameters, which are finally applied to the analysis of the safety performance of the entire structure. The fidelity of the model and the number of discriminative indicators were adjusted in the sensitivity analysis of the individual cable mechanical parameters for the entire structure. By analyzing the influence of varying fidelity and number of discriminative indicators on the accuracy of the analysis results, the relationship between accuracy and the degree of assurance and number of discriminant indicators was obtained, as shown in Figure 8. From the figure, it can be concluded that the fidelity must be within $6 \%$, and the number of discriminative indicators must not be less than 3 to ensure that the discriminative accuracy of sensitivity is above $93.4 \%$. As the model fidelity and number of discriminative indicators increased, the accuracy of the analysis improved significantly, but the analysis accuracy rate of improvement decreased when both increased to a certain level. Therefore, the fidelity of the model used in this study was $5 \%$, and the number of discriminative indicators was three, which met the requirement of the accuracy of the study. 


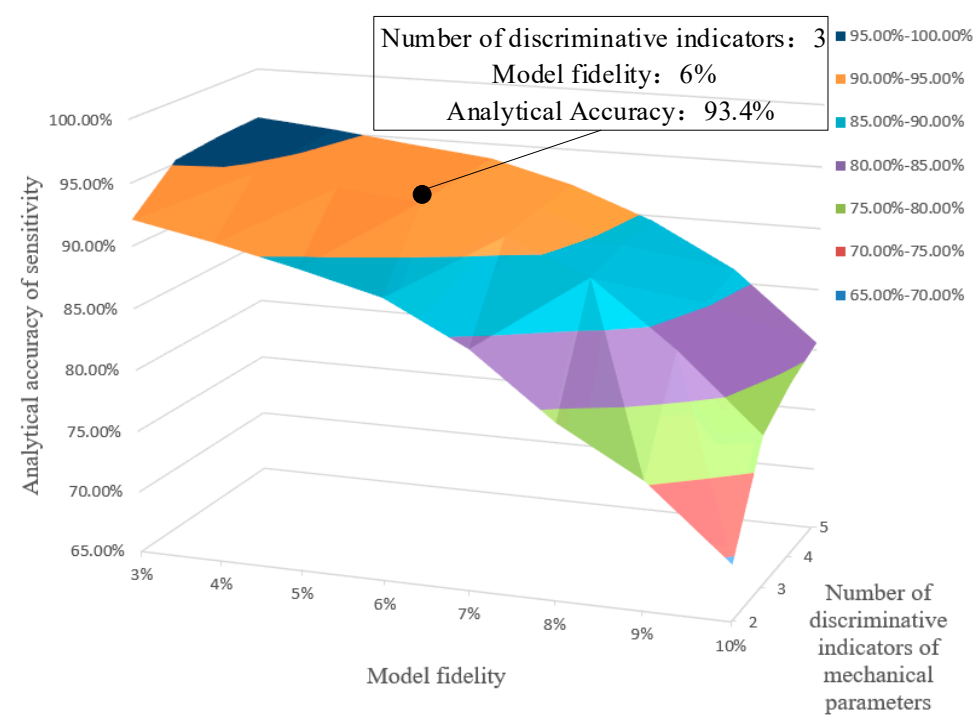

Figure 8. Relationship between the accuracy of sensitive analysis and the fidelity of the model and the number of discriminative indicators.

\section{Discussion and Conclusions}

DT technology is a central to realizing intelligent analysis of structures. A multidimensional model oriented to the intelligent discrimination of structural sensitivity driven by DTs is established, and an analysis method for the intelligent discrimination of prestressed cable sensitivity is proposed by integrating D-S evidence theory to improve the accuracy of structural safety analysis and provide a reliable basis for the maintenance of the structure. In the present study, the following main findings were obtained.

1. Through the capture of monitored and simulated data, a virtual model of the structure with high fidelity can be established using the average weighting method, which provides a model basis for the intelligent analysis of the structure.

2. On the basis of establishing the twin model, the mechanical parameters of the structure are simulated by setting the working conditions. Further, the mechanical properties of each node can be analyzed with high accuracy by integrating multiclass discriminant indices from D-S evidence theory, which guarantees the safety assessment and accurate maintenance of the structure.

3. Through the integration of DTs and D-S evidence theory, the mapping of virtual space to real tension can be realized, and it also provides a reference for the virtual space to control the real tension.

Driven by the DT-based intelligent discrimination method for the sensitivity of the mechanical parameters of prestressed cables, the safety assessment of structures can be carried out by fusing intelligent algorithms with simulated data from twin models, which effectively improves the intelligence and accuracy of structural analysis and also reduces the cost of real-time monitoring of real structures. This overcomes the misjudgment of structural safety performance owing to errors in the data collected by sensors. The change in structural safety performance is related to influencing factors such as the length error of members, wind load, and temperature. In future research, the influence of the change degree of mechanical parameters on the development trend of structural reliability will be focused on according to the concept of DTs and under the comprehensive action of various factors.

Author Contributions: Conceptualization, Z.L.; methodology, Z.L.; software, Z.L.; validation, Z.L., G.S., A.J., and W.L.; writing - original draft preparation, G.S.; writing, review and editing, Z.L.; project administration, Z.L.; funding acquisition, Z.L. All authors have read and agreed to the published version of the manuscript. 
Funding: The research was funded by the National Key R\&D Program for the 13th-Five-Year Plan of China (grant number 2018YFF0300300 The research was funded by the Natural Science Foundation of Beijing, Beijing, China (grant number 8202001).

Institutional Review Board Statement: Not applicable.

Informed Consent Statement: Not applicable.

Data Availability Statement: Data sharing not applicable.

Acknowledgments: The authors would like to thank Beijing University of Technology, Beijing, China, for their support throughout the research project.

Conflicts of Interest: The authors declare no conflict of interest.

\section{References}

1. Kotsovinos, P.; Judge, R.; Walker, G.; Woodburn, P. Fire Performance of Structural Cables: Current Understanding, Knowledge Gaps, and Proposed Research Agenda. J. Struct. Eng. 2020, 146, 03120002. [CrossRef]

2. Krishnan, S. Structural design and behavior of prestressed cable domes. Eng. Struct. 2020, 209, 110294. [CrossRef]

3. Chen, Z.H.; Ma, Q.; Yan, X.Y.; Lou, S.Y.; Chen, R.H.; Si, B. Research on Influence of Construction Error and Controlling Techniques of Compound Cable Dome. J. Hunan Univ. 2018, 45, 47-56. (In Chinese)

4. Guo, Y.L.; Zhang, X.Q. Experimental study on the influences of cable length errors in Geiger cable dome designed with un-adjustable cable length. China Civil. Eng. J. 2018, 51, 52-68. (In Chinese)

5. Zhang, A.L.; Sun, C.; Jiang, Z.Q. Calculation Method of Prestress Distribution for Levy Cable Dome with Double Struts Considering Self-weight. Eng. Mech. 2017, 34, 211-218. (In Chinese)

6. Xue, S.D.; Tian, X.S.; Liu, Y.; Li, X.Y.; Liu, R.J. Mechanical behavior of single-layer saddle-shape crossed cable net without inner-ring. J. Build. Struct. 2021, 42, 30-38. (In Chinese)

7. Liu, Z.S.; Han, Z.B.; He, J.; Wang, Z.Q. Sensitive Test on Relaxation of Cable and Reliability Assessment of Spoke Cable-truss Structure. J. Tongji Univ. 2019, 47, 946-956. (In Chinese)

8. Asadolahi, S.M.; Fanaie, N. Performance of self-centering steel moment frame considering stress relaxation in prestressed cables. Adv. Struct. Eng. 2020, 23, 1813-1822. [CrossRef]

9. Arezki, S.; Kamel, L.; Amar, K. Effects of temperature changes on the behavior of a cable truss system. J. Constr. Steel Res. 2017, 129, 111-118.

10. Castillo, E.; Ramos, A.; Koller, R.; López-Aenlle, M.; Fernández-Canteli, A. A critical comparison of two models for assessmentof atigue data. Int. J. Fatigue 2008, 30, 45-57. [CrossRef]

11. Shekastehband, B.; Abedi, K.; Dianat, N.; Chenaghlou, M.R. Experimental and numerical studies on the collapse behavior of tensegrity systems considering cable rupture and strut collapse with snap-through. Int. J. Non Linear Mech. 2012, 47, 751-768. [CrossRef]

12. Liu, Z.; Shi, G.; Zhang, A.; Huang, C. Intelligent Tensioning Method for Prestressed Cables Based on Digital Twins and Artificial Intelligence. Sensors 2020, 20, 7006. [CrossRef] [PubMed]

13. Greif, T.; Stein, N.; Flath, C.M. Peeking into the void: Digital twins for construction site logistics. Comput. Ind. 2020, 121, 103264. [CrossRef]

14. Zhang, X.H.; Zhang, C.; Wang, M.Y.; Wang, Y.; Du, Y.Y.; Mao, Q.H.; Lv, X.Y. Digital Twin-driven Virtual Control Technology of Cantilever Roadheader.[J/OL]. Comput. Integr. Manuf. Syst. 1-8. Available online: http://kns.cnki.net/kcms/detail/11.5946.TP. 20201026.1618.050.html (accessed on 6 February 2021). (In Chinese).

15. Gong, X.Y.; Lei, K.F.; Wu, Q.Y.; Cui, X.Q.; Wu, Y.; Zhu, B.; Yang, F.Q.; Zhang, H.B.; Liu, H. Digital twin driven airflow intelligent control system for the air outlet of the tunneling face. [J/OL]. China Coal Soc. 2020, 1-10. (In Chinese) [CrossRef]

16. Meng, S.H.; Ye, Y.M.; Yang, Q.; Huang, Z.; Xie, W.H. Digital twin and its aerospace applications. Acta Aeronaut. Astronaut. Sin. 2020, 41, 6-17. (In Chinese)

17. Szpytko, J.; Duarte, Y.S. A digital twins concept model for integrated maintenance: A case study for crane operation. J. Intell. Manuf. 2020, 1-19. [CrossRef]

18. Zhou, Y.; Xing, T.; Song, Y.; Li, Y.; Zhu, X.; Li, G.; Ding, S. Digital-twin-driven geometric optimization of centrifugal impeller with free-form blades for five-axis flank milling. J. Manuf. Syst. 2020. [CrossRef]

19. Yu, J.S.; Song, Y.; Tang, D.Y.; Dai, J. A Digital Twin approach based on nonparametric Bayesian network for complex system health monitoring. J. Manuf. Syst. 2020. [CrossRef]

20. Venkatesan, S.; Manickavasagam, K.; Tengenkai, N.; Vijayalakshmi, N. Health monitoring and prognosis of electric vehicle motor using intelligent-digital twin. IET Electr. Power Appl. 2019, 13, 1328-1335. [CrossRef]

21. Seon, G.; Nikishkov, Y.; Makeev, A.; Ferguson, L. Towards a Digital Twin for Mitigating Void Formation in Autoclave Composite Parts. Eng. Fract. Mech. 2019, 225, 106792. [CrossRef]

22. Angjeliu, G.; Coronelli, D.; Cardani, G. Development of the simulation model for Digital Twin applications in historical masonry buildings: The integration between numerical and experimental reality. Comput. Struct. 2020, 238, 106282. [CrossRef] 
23. Hu, Z.Z.; Tian, P.L.; Li, S.W.; Zhang, J.P. BIM-based integrated delivery technologies for intelligent MEP management in the operation and maintenance phase. Adv. Eng. Softw. 2018, 115, 1-16. [CrossRef]

24. Stepinac, M.; Gašparović, M. A Review of Emerging Technologies for an Assessment of Safety and Seismic Vulnerability and Damage Detection of Existing Masonry Structures. Appl. Sci. 2020, 10, 5060. [CrossRef]

25. Chen, W.T.; Tsai, I.C.; Merrett, H.C.; Lu, S.T.; Lee, Y.I.; You, J.K.; Mortis, L. Construction Safety Success Factors: A Taiwanese Case Study. Sustainability 2020, 12, 6326. [CrossRef]

26. Li, G.Q.; Cao, K.; Lu, Y.; Jiang, J. Effective length factor of columns in non-sway modular steel buildings. Adv. Steel Constr. 2017, $13,412-426$.

27. Lacey, A.W.; Chen, W.; Hao, H.; Bi, K. Effect of inter-module connection stiffness on structural response of a modular steel building subjected to wind and earthquake load. Eng. Struct. 2020, 213, 110628. [CrossRef]

28. Chen, W.; Ye, J.H.; Jin, L.; Jiang, J.; Liu, K.; Zhang, M.; Chen, W.W.; Zhang, H. High-temperature material degradation of Q345 cold-formed steel during full-range compartment fires. J. Constr. Steel Res. 2020, 175, 106366. [CrossRef]

29. Okazaki, S.; Okuma, C.; Kurumatani, M.; Yoshida, H.; Matsushima, M. Predicting the Width of Corrosion-Induced Cracks in Reinforced Concrete Using a Damage Model Based on Fracture Mechanics. Appl. Sci. 2020, 10, 5272. [CrossRef]

30. Zeng, B.; Zhou, Z.; Zhang, Q.F.; Xu, Q.; Meng, S.P. Analytical and experimental research on damage identification of cable-stayed arch-truss based on data fusion. China Civil. Eng. J. 2020, 53, 28-37+86. (In Chinese)

31. Bappy, M.M.; Ali, S.M.; Kabir, G.; Paul, S.K. Supply chain sustainability assessment with Dempster-Shafer evidence theory: Implications in cleaner production. J. Clean. Prod. 2019, 237, 117771. [CrossRef]

32. Denoeux, T.; Shenoy, P. An Interval-Valued Utility Theory for Decision Making with Dempster-Shafer Belief Functions. Int. J. Approx. Reason. 2020, 124, 194-216. [CrossRef] 